\title{
CHANDRA X-RAY OBSERVATIONS OF THE HYDRA A CLUSTER: AN INTERACTION BETWEEN THE RADIO SOURCE AND THE X-RAY-EMITTING GAS
}

\author{
B. R. McNamara, ${ }^{1}$ M. Wise, ${ }^{3}$ P. E. J. Nulsen,,${ }^{1,2}$ L. P. David,${ }^{1}$ C. L. Sarazin, ${ }^{4}$ M. Bautz,${ }^{3}$ \\ M. MARKeVitch,${ }^{1}$ A. Vikhlinin, ${ }^{1}$ W. R. Forman,${ }^{1}$ C. Jones, ${ }^{1}$ D. E. HARris,${ }^{1}$
}

submitted to The Astrophysical Journal Letters

\begin{abstract}
We present Chandra X-ray Observations of the Hydra A cluster of galaxies, and we report the discovery of structure in the central $80 \mathrm{kpc}$ of the cluster's X-ray-emitting gas. The most remarkable structures are depressions in the X-ray surface brightness, $\sim 25-35 \mathrm{kpc}$ diameter, that are coincident with Hydra A's radio lobes. The depressions are nearly devoid of X-ray-emitting gas, and there is no evidence for shock-heated gas surrounding the radio lobes. We suggest the gas within the surface brightness depressions was displaced as the radio lobes expanded subsonically, leaving cavities in the hot atmosphere. The gas temperature declines from $4 \mathrm{keV}$ at $70 \mathrm{kpc}$ to $3 \mathrm{keV}$ in the inner $20 \mathrm{kpc}$ of the brightest cluster galaxy (BCG), and the cooling time of the gas is $\sim 600 \mathrm{Myr}$ in the inner $10 \mathrm{kpc}$. These properties are consistent with the presence of a $\sim 34 \mathrm{M}_{\odot} \mathrm{yr}^{-1}$ cooling flow within a 70 $\mathrm{kpc}$ radius. Bright $\mathrm{X}$-ray emission is present in the $\mathrm{BCG}$ surrounding a recently-accreted disk of nebular emission and young stars. The star formation rate is commensurate with the cooling rate of the hot gas within the volume of the disk, although the sink for the material cooling at larger radii remains elusive. A bright, unresolved X-ray source is present in the BCG's nucleus, coincident with the radio core. It's X-ray spectrum is consistent with a power law absorbed by a foreground $N_{\mathrm{H}} \simeq 4 \times 10^{22} \mathrm{~cm}^{-2}$ column of hydrogen. This column is roughly consistent with the hydrogen column seen in absorption toward the $\lesssim 24$ pc diameter VLBA radio source. Apart from the point source, no evidence for excess X-ray absorption above the Galactic column is found.
\end{abstract}

Subject headings: Galaxies: clusters: individual (Hydra A) - cooling flows - intracluster medium

\section{INTRODUCTION}

The Hydra A radio galaxy is associated with a relatively poor cluster of galaxies at redshift $z=0.052$. The cluster harbors an atmosphere of X-ray emitting gas of luminosity $L_{x}(0.5-4.5)=2.2 \times 10^{44} \mathrm{ergs} \mathrm{s}^{-1}$, and a mean gas temperature of $\sim 4 \mathrm{keV}$ based on Einstein MPC observations (David et al. 1990) and ASCA observations (Ikebe et al. 1997). A cooling flow is present with an accretion rate of $\dot{M} \sim 250 \mathrm{M}_{\odot} \mathrm{yr}^{-1}$ (assuming $\mathrm{H}_{0}=50 \mathrm{~km} \mathrm{~s}^{-1} \mathrm{Mpc}^{-1}$ ) within a radius of $\simeq 170$ kpc (White et al. 1997; Peres et al. 1998). The hot atmosphere is centered on a brightest cluster elliptical galaxy (BCG) that hosts a large ( $80 \operatorname{arcsec}$ or $84 \mathrm{kpc}$ in projection), unusually powerful $\left(P=1.6 \times 10^{26} \mathrm{~W} \mathrm{~Hz}^{-1}\right.$ at $\left.178 \mathrm{MHz}\right)$ Fanaroff-Riley type 1 (FR 1) radio source 3C 218 (Ekers \& Simkin 1983; Taylor et al. 1990; Taylor 1996). The twin jet-lobe radio source emerges from a disk of young stars (McNamara 1995; Hansen et al. 1995; Melnick et al. 1997) and nebular emission (Ekers \& Simkin 1983; Hansen et al. 1995; Baum et al. 1988) several $\mathrm{kpc}$ in size that is in rotation about the nucleus. H I is seen in absorption toward the nucleus (Dwarakanath, Owen, \& van Gorkom 1995) and has been mapped in absorption against the parsec scale nuclear radio source with the VLBA (Taylor 1996).

Hydra A was observed by the Chandra X-ray Observatory during its orbital verification and activation phase. Hydra A's several interesting properties provide a first opportunity to investigate a cooling flow and potential interactions between

${ }^{1}$ Harvard-Smithsonian Center for Astrophysics, 60 Garden St. Cambridge, MA 02138

${ }^{2}$ Department of Engineering Physics, University of Wollongong, Wollongong NSW 2522, Australia

${ }^{3}$ Massachusetts Institute of Technology, Center for Space Research, Cambridge, MA 02139

${ }^{4}$ Astronomy Department, University of Virginia, Charlottesville, VA the radio source and X-ray-emitting gas using Chandra's unprecedentedly high spatial resolution. We present an analysis of Hydra A based on the preliminary telescope calibration in this Letter, and report the discovery of an interaction between the radio source and the $\mathrm{X}$-ray-emitting gas. We assume $\mathrm{H}_{0}=70 \mathrm{~km} \mathrm{~s}^{-1} \mathrm{Mpc}^{-1}, \Omega_{\mathrm{M}}=0.3, \Omega_{\Lambda}=0.7$, a luminosity distance of $240 \mathrm{Mpc}$, and 1 arcsec $=1.05 \mathrm{kpc}$.

\section{DATA ANALYSIS}

The calibration observations of Hydra A were performed on 1999 October 30. A total integration time of $40 \mathrm{ksec}$ was obtained, $20 \mathrm{ksec}$ centered at the aim point of the S3 backilluminated ACIS chip (OBSID 576), and $20 \mathrm{ksec}$ at the ACIS I aim point on the I3 front-illuminated device (OBSID 575). $\mathrm{X}$-ray events with energies below $300 \mathrm{eV}$ and above $10 \mathrm{keV}$ were not considered in our analysis, and flight grades $0,2,3$, 4,6 were retained. The particle background was generally stable throughout the S3 observations. Only 300s of the $20 \mathrm{ksec}$ exposure experienced a $30 \%$ increase in particle background. Therefore no S3 data were rejected on this basis. The spectral analysis presented here is limited to a $0.5-7 \mathrm{keV}$ bandpass for the S3 data.

\subsection{X-ray Morphology}

A Chandra image of the X-ray emission from the central $118 \times 118$ arcsec of the cluster is shown in Fig. 1. The image is a summed, $40 \mathrm{ksec}$ exposure obtained with the ACISI3, front illuminated device, and the ACIS-S3, back-illuminated device. The top panel shows the unsmoothed image, after event filtering, centered on the X-ray point source. The X-ray emission shows a great deal of structure on scales ranging from less than a few kpc to tens of kpc that have not been seen in ear- 
lier X-ray imagery. An X-ray point source $(R A=091805.77$ , Dec $=-120542.53, \mathrm{~J} 2000)$, shown inset to the top panel of Fig. 1, coincides with the central radio core and BCG nucleus. The lower panel of Fig. 1 shows the wavelet-smoothed X-ray image. The emission is concentrated in a central triangular region, 10-15 arcsec in size, and in fainter fingers of emission extending northeast and southwest of the center. The emission spectrum of this material is consistent with thermal emission from $\sim 3 \mathrm{keV}$ cluster gas. Two depressions in the X-ray emission, 20-30 arcsec in diameter, are seen $\sim 20$ arcsec to the north and south of the cluster center. The surface brightness within these cavities is a factor of $\sim 1.5$ lower than the mean surface brightness of the emission at similar radii from the center. This decline in surface brightness is consistent with these regions being devoid of gas at the ambient temperature and density.

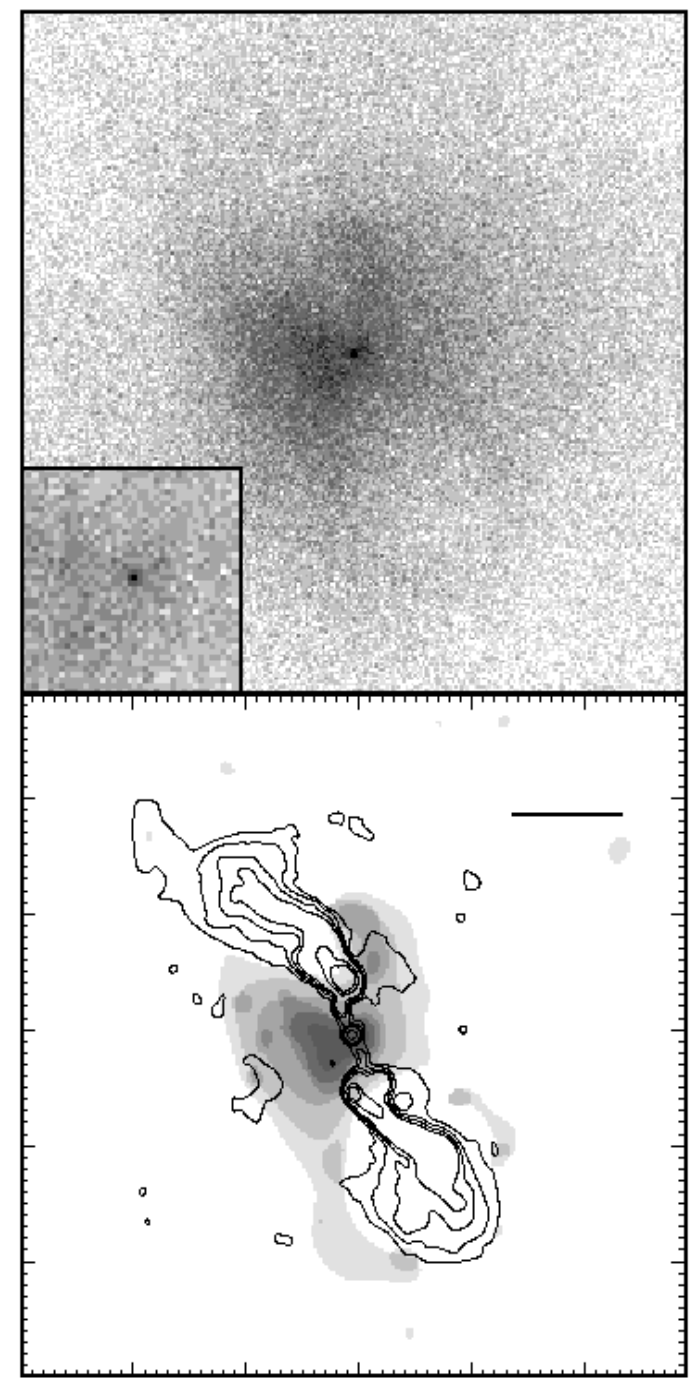

FIG. 1. Top Panel: $40 \mathrm{ksec}$ integration X-ray image of the central region of the Hydra A cluster centered on the nuclear X-ray point source (shown inset). Bottom Panel: Wavelet smoothed and reconstructed image of the same region superposed on the $6 \mathrm{~cm}$ VLA Radio image of Hydra A (contours). The scale bar is 20 arcsec in length.

\subsection{Radio Morphology}

A $6 \mathrm{~cm}$ VLA image obtained in the A-array, obtained by G. Taylor, is shown superposed on the wavelet-reconstructed Xray image in Fig. 1. The cavities in the X-ray emission are filled by emission from the radio lobes, and the X-ray cavities and radio lobes are remarkably similar in shape. Similar cavities have been seen in ROSAT imagery of the Perseus cluster (Böhringer et al. 1992) and in Cygnus A (Carilli et al. 1994). Heinz et al. (1998) proposed a model to explain such cavities as a shell of shocked gas displaced by the relativistic material from the radio jet. This model would imply that the X-ray emission surrounding the radio source should be considerably hotter than the material away from the radio source. We evaluate this interpretation of the radio cavities, and we explore the physical state of the cluster gas in $\$ 2.4$.

\subsection{Optical Morphology: The Central Disk}

Spatial correlations between the X-ray, optical, and radio emission are present in the inner $20 \mathrm{kpc}$ of the BCG. The $U$ band contours are superposed on a grayscale X-ray image in Fig. 2. The X-ray point source is centered on a gaseous disk of nebular emission and young blue stars in rotation about the nucleus (Ekers \& Simkin 1983; Baum et al. 1988; McNamara 1995; Hansen et al. 1995; Melnick et al. 1997). The disk of star formation is about $10 \mathrm{kpc}$ by $7 \mathrm{kpc}$ in size, and is roughly 0.7 magnitudes bluer than the $U-I$ color of the galaxy's halo (McNamara 1995). The nebular emission (Baum et al. 1988; Hansen et al. 1995) extends over a similar region.

The brightest X-ray emission, apart from the point source, appears in a flattened structure coincident with the disk, and in an irregular structure several arcsec east of the disk. The Xray point source is within a half arcsec of the radio core (Fig. 1) and the BCG's nucleus. This positional correspondence is within Chandra's absolute pointing error. The surface brightness of the light gray region in Fig. 2 is $\simeq 30 \%$ fainter than the dark gray structure just east of the disk. This fainter emission forms a wedge-shaped structure that widens toward two companion galaxies projected onto the BCG (see also Fig. 1). The asymmetry in the X-ray structure may be caused, in part, by an irregular potential well associated with a merger between the BCG and the companion galaxies (Ekers \& Simkin 1983).

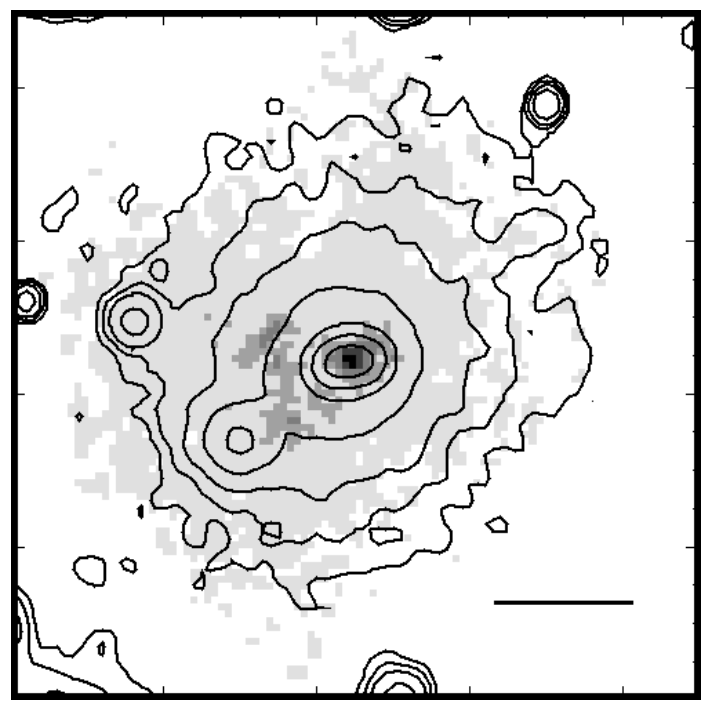

FIG. 2. Detail of the X-ray emission, grayscale smoothed with a $F W H M=1.75$ pix gaussian, superposed on the $U$-band contours of the BCG (McNamara 1995). The central disk of gas and young stars are the elliptical contours centered on the X-ray point source. The scale bar is 10 arcsec in length.

The radio jets (Fig. 1) emerge from the optical disk at roughly a $20 \mathrm{deg}$ angle from the disk's minor optical axis and 
rotation axis, and flare into lobes where the gas pressure reaches $\sim 6 \times 10^{-10} \mathrm{erg} \mathrm{cm}^{-3}$. The jets show no obvious signs of interacting with the optical disk emission or the X-ray emission in the central flattened structure.

\subsection{Physical State of the X-ray Emitting Gas}

We computed the radial distribution of density, temperature, and pressure of the gas in the central $84 \mathrm{kpc}$. These parameters were computed by fitting a single temperature XSPEC model to the X-ray emission in annuli centered on a central X-ray point source. The fluxes were corrected for cluster emission at large radii projected onto the inner regions (deprojected), and the abundance and foreground absorption were free parameters in the models. The abundances were found to be $\sim 0.4$ solar, and the foreground absorption $\sim 2 \times 10^{20} \mathrm{~cm}^{-2}$. No evidence for excess absorption from cold gas within the cluster is found, with the exception of a large column toward the nuclear point source (\$4). In addition, we constructed hardness ratio profiles, $\kappa(R)$, by taking the ratios of the X-ray surface brightnesses, $I$, in several passbands. These profiles are plotted in Fig. 3. The hardness ratios are defined as $\kappa_{1}=I(0.5 \rightarrow 1.5) / I(1.5 \rightarrow 2.5)$ (open symbols) and $\kappa_{2}=I(0.5 \rightarrow 2.5) / I(2.5 \rightarrow 6.0)$ (filled symbols), where the figures in brackets are the passbands in $\mathrm{keV}$. The spectrum is harder as $\kappa(R)$ decreases. The $n_{e}, k T$, and $P$ profiles exclude the central point source, while the hard (absorbed) point source is included in the hardness ratio plot.

The temperature increases from $\sim 3 \mathrm{keV}$ in the central $10 \mathrm{kpc}$ to $\sim 4 \mathrm{keV}$ at $80 \mathrm{kpc}$. The rms density within the central $10 \mathrm{kpc}$ is $n_{e}=0.06 \mathrm{~cm}^{-3}$. The density declines with radius as $\sim r^{-1}$ to a radius of $70 \mathrm{kpc}$, and the gas pressure declines by a factor of $\sim 6$ from the center to $70 \mathrm{kpc}$. The central point source is considerably harder than the surrounding emission within a 10 $\mathrm{kpc}$ radius. Beyond the central source, $\kappa$ decreases (hardens) $10 \%-30 \%$ between the center and $80 \mathrm{kpc}$. The broad-band $\kappa_{2}$ shows the larger decline. There is no significant difference between hardness ratio profiles including or excluding the flux from the cavities.

We note that in the $0.5-7 \mathrm{keV}$ Chandra band, the count rate for gas at $80 \mathrm{keV}$ is about $2 / 3$ of that for gas with the same emission measure at $5 \mathrm{keV}$. If gas in or near the cavities has been compressed in a shock, its emission in the Chandra band would be larger than from the same amount of gas at ambient conditions. Then the regions within the radio lobes would be brighter than the surrounding emission, which is not observed.

\section{DISCUSSION}

\subsection{Origin of the $X$-ray Cavities}

There is no indication that the gas surrounding the radio source is hotter than the ambient cluster gas. This behavior is inconsistent with strongly shock heated gas, as is suggested by the Heinz et al. (1998) model, but is consistent with the cool central material being displaced as the radio source expands subsonically. There is no evidence that the radio source is heating the gas outside the cavities (Nulsen et al. in preparation).

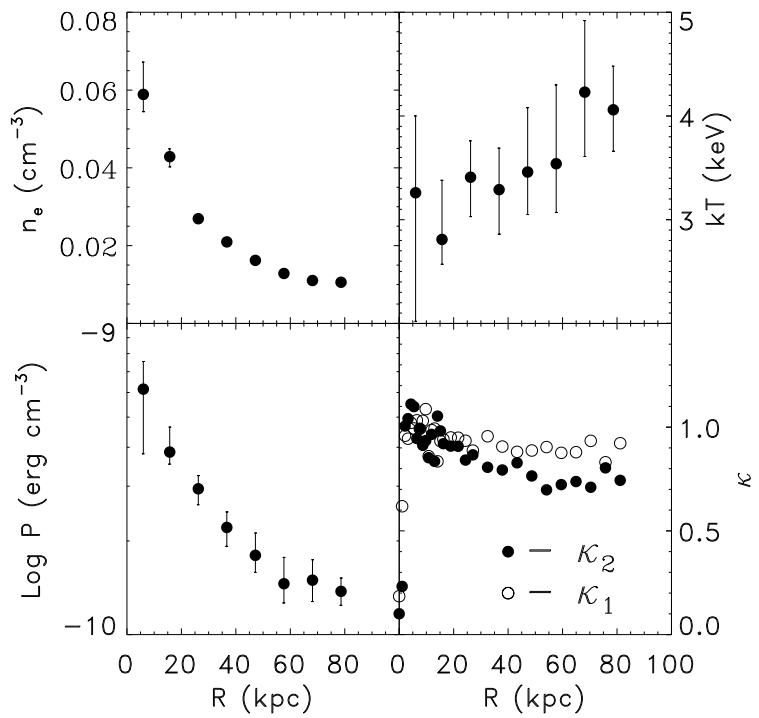

FIG. 3. Run of electron density (upper left), temperature (upper right), pressure (lower left), and hardness ratio (lower right). In this convention, hardness increases as $\kappa$ decreases.

We now calculate the minimum energy required by the radio source to displace the gas using the southern cavity for illustration. The center of the southern cavity is approximately 26 arcsec from the X-ray nuclear point source. The electron density in the annulus centered on the southern cavity is $n_{\mathrm{e}}=0.027 \mathrm{~cm}^{-3}$ and the temperature is $k T=3.4 \mathrm{keV}$, giving a pressure $p=2.8 \times 10^{-10} \mathrm{erg} \mathrm{cm}^{-3}$. Based on the deprojection, undisturbed gas in a volume the size of this cavity would have contributed about 2000 counts. This is consistent with our estimate for the count deficit in the cavity. We therefore assume that the ambient ICM has been displaced from this region by the radio source. Since there is no spectral hardening at the edges of the cavities, we assume that the pressure within them is similar to the ambient gas pressure.

The minimum energy required to push the gas out of a sphere of radius $15 \mathrm{kpc}$ is then $p V \simeq 1.2 \times 10^{59} \mathrm{erg}$. Since there are no signs of shocks, we assume that gas motions are subsonic and can be ignored, so that the total energy required to inflate the cavity is a modest multiple of this.

The cavity would take $\sim 2 \times 10^{7}$ yr to expand at approximately the sound speed. The cavity is also buoyant, so it must be refilled on a similar timescale, $\simeq 2 R \sqrt{r / G M(R)}$, where $r$ is the radius of the cavity and $R$ is the distance to the cluster center. From the deprojection, a rough estimate of the total gravitating mass within $30 \mathrm{kpc}$ of the cluster center is $M(R)=3 \times 10^{12} \mathrm{M}_{\odot}$, giving a refilling time of about $6 \times 10^{7} \mathrm{yr}$. Thus, the minimum mechanical power from the southern radio jet required to maintain the cavity is $\sim 6 \times 10^{43} \mathrm{erg} \mathrm{s}^{-1}$, which is comparable to the total radio power from Hydra A (Ekers \& Simkin 1983). Either timescale implies an efficiency of conversion of kinetic energy to radio power of order unity, and we need not postulate the existence of kinetic luminosity substantially in excess of the luminous radio power (e.g. Heinz et al. 1998).

Our interpretation requires the radio lobes and surrounding gas to be nearly in pressure equilibrium. However, the gas pressure appears to be more than an order of magnitude larger than the minimum energy pressure of the radio lobes (Taylor et al. 1990). If the lobes are in pressure balance with the cluster gas, and if equipartition between the magnetic field and relativistic 
particles is to be maintained, then one or more of the following conditions is implied. There is a significant additional contribution to the particle energy density from low energy electrons or from protons (e.g. Böhringer et al. 1993), the radio filling factior is significantly less than one, or the radio lobes are at significantly larger radii in the cluster atmosphere than their projected positions.

\subsection{The Cooling Flow}

Gas with the observed central density $n_{e}(r<10 \mathrm{kpc})=$ $0.06 \mathrm{~cm}^{-3}$, temperature $k T=3 \mathrm{keV}$, and abundance of 0.4 solar has a radiative cooling time of $\sim 6 \times 10^{8} \mathrm{yr}$. If such cool material has been present in the cluster for $\gtrsim 1 \mathrm{Gyr}$, the gas should cool to low temperatures and flow to the center of the cluster. The Chandra data allow us to obtain high quality spectra of several independent regions within the central $80 \mathrm{kpc}$ of the cluster. We have fitted an absorbed thermal (mekal) plus constant pressure cooling flow (mkcflow) model to a series of 8 circular apertures ranging in equal steps from $10 \operatorname{arcsec}$ to $80 \operatorname{arcsec}$ in radius. The central 1.5 arcsec was excluded. Assuming spherical symmetry, we attribute the differences in mass deposition rate, $\dot{M}$, for successive apertures to the annulus they define, apply a standard geometric deprojection to convert these to mass deposition rates per unit volume, and calculate total $\dot{M}$ 's for the spheres. The total mass deposition rate within a $74 \mathrm{kpc}$ sphere is $34 \pm 5 \mathrm{M}_{\odot} \mathrm{yr}^{-1}$, and $\dot{M}(R) \sim R^{1.1}$.

\subsection{Cooling Rate vs Star Formation Rate}

The most controversial issue concerning cooling flows is the fate of the cooling material. We now ask whether the accretion rates derived from the new X-ray data are consistent with the star formation rate. Star formation appears to be confined to the disk (\$2.3), whose young stellar population mass estimate ranges from $10^{7.7 \rightarrow 9} \mathrm{M}_{\odot}$ (McNamara 1995; Hansen et al. 1995). Estimates of the average star formation rates vary from $\lesssim 1 \mathrm{M}_{\odot} \mathrm{yr}^{-1}$ for continuous star formation for $\sim \mathrm{Gyr}$ duration, to $\sim 15 \mathrm{M}_{\odot} \mathrm{yr}^{-1}$ for a younger, $\sim 10^{8} \mathrm{yr}$ old burst population. The degree to which star formation may be consuming the cooling gas depends on factors including the relative ages of the cooling flow and the stellar population, and the stellar initial mass function. In the central $10 \mathrm{kpc}$, a region somewhat larger than the central optical disk, the mass deposition rate is $4 \pm 2 \mathrm{M}_{\odot} \mathrm{yr}^{-1}$. Thus, the observed rates of star formation can account entirely for the mass cooling within this region regardless of the age of the cooling flow. However, the disk star formation would provide a sink for the cooling gas within the entire $74 \mathrm{kpc}$ region for $\lesssim 3 \times 10^{6} \mathrm{yr}$, which is much smaller than the minimum time $\sim 1$ Gyr required to establish a cooling flow. Most of the cooling material remains unaccounted for, and there is no evidence that the radio source is heating the gas and reducing the cooling rates.

\section{THE CENTRAL POINT SOURCE}

We extracted the spectrum of the central point source within a 1 arcsec radius aperture. The background was taken from the annular region between $r=1-2.5$ arcsec centered on the point source. The net point source spectrum was found by subtracting the background spectrum, after normalizing by the relative areas of the central and annular regions. The net spectrum appears to be a power law absorbed by a $N_{\mathrm{H}}=4.5(2.2-9.9) \times$ $10^{22} \mathrm{~cm}^{-2}$ (90\% errors). The point source flux, uncorrected for absorption, is $f(0.6-6.0)=1.0 \times 10^{-13} \mathrm{ergs} \mathrm{s}^{-1} \mathrm{~cm}^{-2}$, which corresponds to a luminosity of $6.9 \times 10^{41} \mathrm{ergs} \mathrm{s}^{-1}$. The absorbing column toward the point source is more than two orders of magnitude larger than the Galactic foreground column, and is confined to a spatial extent of $<1.5$ arcsec.

This column density is in reasonably good agreement with the column of neutral hydrogen absorption seen toward the VLBI radio source in the nucleus of the galaxy (Taylor 1996). The radio absorption map confines the spatial extent of this high column density material to a region of $\lesssim 24$ pc in the nucleus of the galaxy. Assuming the X-ray and radio flux is absorbed by the same material, the combined observations restrict the $\mathrm{X}$ ray-emitting region of the point source to $\lesssim 24$ pc.

\section{ACKNOWLEDGMENTS}

PEJN gratefully acknowledges the hospitality of the Harvard-Smithsonian Center for Astrophysics. BRM acknowledges grant NAS8-39073.

\section{REFERENCES}

Baum, S.A., Heckman, T., Bridle, A., van Breugel, W., \& Miley, G., 1988, ApJS, 68, 643

Böhringer, H., Voges, W., Fabian, A.C., Edge, A.C., \& Neumann, D.M. 1993, MNRAS, 264, L25

Carilli, C.L., Perley, R.A., \& Harris, D.E. 1994, MNRAS, 270, 173

David, L.P., Arnaud, K.A., Forman, W., \& Jones, C. 1990, ApJ, 356,32

Dwarakanath, K.S., Owen, F.N., \& van Gorkom, J.H., 1995, ApJ, 442, L1

Ekers, R.D., \& Simkin, S.M. 1983, ApJ, 265, 85

Hansen, L., Jørgenson, H.E., \& Nørgaard-Nielson, H.U. 1995, AA, 297, 13

Heinz, S., Reynolds, C.S., \& Begelman, M.C. 1998, ApJ, 501, 126

Ikebe, Y., Makishima, K., Ezawa, H., Fukazawa, Y., Hirayama, M., Honda, H., Ishisaki, Y., Kikuchi, K., Kubo, H., Murakami, T., Ohashi, T., Takahashi, T., \& Yamashita, K. 1997, ApJ, 481, 660

McNamara, B.R. 1995, ApJ, 443, 77

Melnick, J., Gopal-Krishna, \& Terlevich, R. 1997, AA, 318, 337

Peres, C. B., Fabian, A. C., Edge, A. C., Allen, S. W., Johnstone, R. M., \& White, D. A. 1998, MNRAS, 298, 416

Taylor, G.B. 1996, ApJ, 470, 394

Taylor, G.B., Perley, R.A., Inoue, M., Kato, T., Tabara, H., \& Aizu, K., 1990, ApJ, 360, 41

White, D. A., Jones, C., \& Forman, W. 1997, MNRAS, 292, 419 